AperTO - Archivio Istituzionale Open Access dell'Università di Torino

\title{
Dynamics, stability and iron-binding activity of frataxin clinical mutants
}

\section{This is the author's manuscript}

Original Citation:

Availability:

This version is available http://hdl.handle.net/2318/1661474

since 2018-03-06T17:34:33Z

Published version:

DOI:10.1111/j.1742-4658.2008.06512.x

Terms of use:

Open Access

Anyone can freely access the full text of works made available as "Open Access". Works made available under a Creative Commons license can be used according to the terms and conditions of said license. Use of all other works requires consent of the right holder (author or publisher) if not exempted from copyright protection by the applicable law. 
This is the author's final version of the contribution published as:

Dynamics, stability and iron-binding activity of frataxin clinical mutants.

Correia AR, Pastore C, Adinolfi S, Pastore A, Gomes CM.

FEBS J. 2008 Jul;275(14):3680-90.

The publisher's version is available at:

doi: 10.1111/j.1742-4658.2008.06512.x

When citing, please refer to the published version. 


\title{
Dynamics, stability and iron-binding activity of frataxin clinical mutants
}

\author{
Correia AR, Pastore C, Adinolfi S, Pastore A, Gomes CM.
}

\begin{abstract}
Friedreich's ataxia results from a deficiency in the mitochondrial protein frataxin, which carries single point mutations in some patients. In the present study, we analysed the consequences of different disease-related mutations in vitro on the stability and dynamics of human frataxin. Two of the mutations, G130V and D122Y, were investigated for the first time. Analysis by CD spectroscopy demonstrated a substantial decrease in the thermodynamic stability of the variants during chemical and thermal unfolding (wild-type $>$ W155R $>$ I154F $>$ D122Y $>$ G130V), which was reversible in all cases. Protein dynamics was studied in detail and revealed that the mutants have distinct propensities towards aggregation. It was observed that the mutants have increased correlation times and different relative ratios between soluble and insoluble/aggregated protein. NMR showed that the clinical mutants retained a compact and relatively rigid globular core despite their decreased stabilities. Limited proteolysis assays coupled with LC-MS allowed the identification of particularly flexible regions in the mutants; interestingly, these regions included those involved in iron-binding. In agreement, the iron metallochaperone activity of the Friedreich's ataxia mutants was affected: some mutants precipitate upon iron binding (I154F and W155R) and others have a lower binding stoichiometry (G130V and D122Y). Our results suggest that, in heterozygous patients, the development of Friedreich's ataxia may result from a combination of reduced efficiency of protein folding and accelerated degradation in vivo, leading to lower than normal concentrations of frataxin. This hypothesis also suggests that, although quite different from other neurodegenerative diseases involving toxic aggregation, Friedreich's ataxia could also be linked to a process of protein misfolding due to specific destabilization of frataxin.
\end{abstract}

\footnotetext{
Abbreviations

FRDA

Friedreich's ataxia

GST

glutathione $S$-transferase

HSQC

heteronuclear single quantum coherence

$T_{1}$

longitudinal relaxation rate

$T_{2}$

transverse relaxation rate

$\tau_{\mathrm{c}}$

correlation time
} 
Human frataxin is a mitochondrial protein whose deficiency is associated with the neurodegenerative disorder Friedreich ataxia (FRDA; OMIM 229300), a pathology characterized by neuronal death, cardiomyopathy and diabetes [1]. At the molecular level, the disease involves iron homeostasis deregulation and an impairment of the biosynthesis of iron-sulfur proteins [1-4]. The majority of FRDA patients (> 95\%) are homozygous for a GAA repeat expansion within the first intron of the frataxin gene [5,6]. The expansion affects frataxin transcription, which results in a reduction of protein levels by $5-35 \%$, depending on the insertion length. A small but significant number of FRDA patients are compound heterozygotes, containing a GAA expansion in one allele and a point mutation in the other [7]. About 15 distinct point mutations are currently known [7,8] and, although some account for atypical clinical presentations, no clear correlation can be made considering the lower number of patients characterized.

In preliminary studies, we addressed the question of whether prevalent mutations that result in classical FRDA phenotypes were correlated with complete impairment of the frataxin fold [9]. We showed that, although destabilized, the two tested mutations (W155R and I154F) result in proteins that should be folded under physiological conditions. What then is the pathogenic mechanism? Two possible working hypotheses are that, in the mutants, the efficiency of folding is reduced compared to that of the wild-type protein and/or that the mutants have an enhanced susceptibility to degradation. Either scenario or a combination of both, is likely to lead to lower than normal frataxin concentrations. To address this important question, which bears direct relevance for our understanding of FRDA, we performed a comparative study of the protein dynamics of frataxin variants carrying mutations of clinical interest. We focused on how the frataxin mutations I154F, W155R, D122Y and G130V encompass structural perturbations that may compromise proteinprotein interactions [10-12], impair functional activity (in terms of iron binding and metallochaperone activity) [4] and increase post-translational proteolytic susceptibility. We also addressed in detail how mutations affect the protein dynamics. The study approach is expected to contribute to a better molecular and structural understanding of the disease mechanism, especially when taken in combination with recent data obtained in vivo in human cells for some of these mutations [10].

\section{Results}

\section{Mapping frataxin mutations on the structure}

The four mutations D122Y, G130V, I154F and W155R were mapped onto the human frataxin structure (Fig. 1). Three of them are replacements of exposed residues. The mutation D122Y is located at the very beginning of the $\beta 1$ strand and is an integral part of the turn connecting $\alpha 1$ to $\beta 1$. The side chain of D122 could potentially form an H-bond with the amide group of the spatially contiguous G138. Being in a turn, the exact nature of this residue could influence the folding process. Furthermore, a stabilizing surface ionic interaction of D122 with the nearby K135 residue is disrupted upon mutation. Similarly, G130 is in the tight turn formed by G128, S129 and G130 between strands $\beta 1$ and $\beta 2$ and both $\varphi$ and $\psi$ are positive. Its mutation into a valine must disturb the turn conformation, resulting in severe local strain. I154 is a buried residue that directly sits into the hydrophobic core; its replacement by another, albeit bulkier, but still hydrophobic residue does not disrupt the fold completely [9]. Finally, W155 is an exposed and extremely conserved residue that has been suggested to be relevant for protein-protein interactions. However, because W155 packs against a nearby arginine (R165), its mutation to an arginine results in a repulsive interaction arising from two spatially contiguous positively charged residues. 


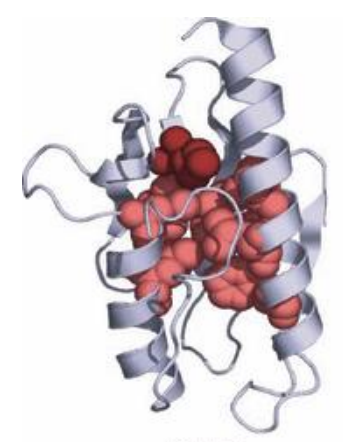

I154F

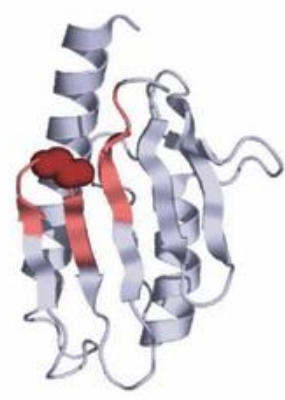

G130V

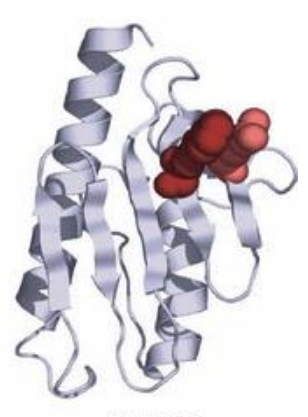

W155R

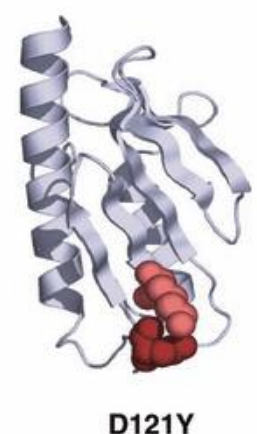

Figure 1.

Frataxin mutations involved in Friedreich's ataxia. Figures were drawn using the protein databank file 1EKG.

\section{Protein dynamics of wild-type human frataxin}

The dynamical properties of wild-type human frataxin were established by NMR ${ }^{15} \mathrm{~N}$ relaxation experiments, looking specifically at regions around the mutated positions (Fig. 2A; see also supplementary Figs S1-S3). This technique has proven to be very successful in providing information about molecular internal motions. Overall, longitudinal $\left(T_{1}\right)$ and transverse $\left(T_{2}\right)$ relaxation rates and NOE values are rather uniform along the protein sequence, in agreement with what is expected for a compactly folded globular protein. Such a flat behaviour is consistent with the presence of only short and rather stiff turns between secondary structure elements. The LipariSzabo model-free formalism was used to analyse the data [13]. Smaller than average $T_{1} / T_{2}$ and small or negative NOE values, which suggest the presence of internal motions on the nano- and picosecond timescale, were observed at both termini and especially at the $\mathrm{C}$-terminus. This suggests a higher mobility of these regions compared to the rest of the molecule, in agreement with the larger rmsd of the solution bundle in these regions [14]. Residues in the loop between strands $\beta 4$ and $\beta 5$ (Thr149, Asn151 and Lys152), and at the end of strand $\beta 6$ (Val174), have larger than average $T_{1} / T_{2}$ ratios and shorter $\mathrm{T}_{2}$ (see supplementary Figs S1-S3). These features may indicate the presence of low-frequency motions, often associated with conformational exchange. The correlation time of the wild-type at room temperature, as estimated from the $T_{1} / T_{2}$ ratio, is $7.9 \mathrm{~ns}$. This value is in good agreement with the value expected for a monomeric globular domain of equivalent size [14]. 

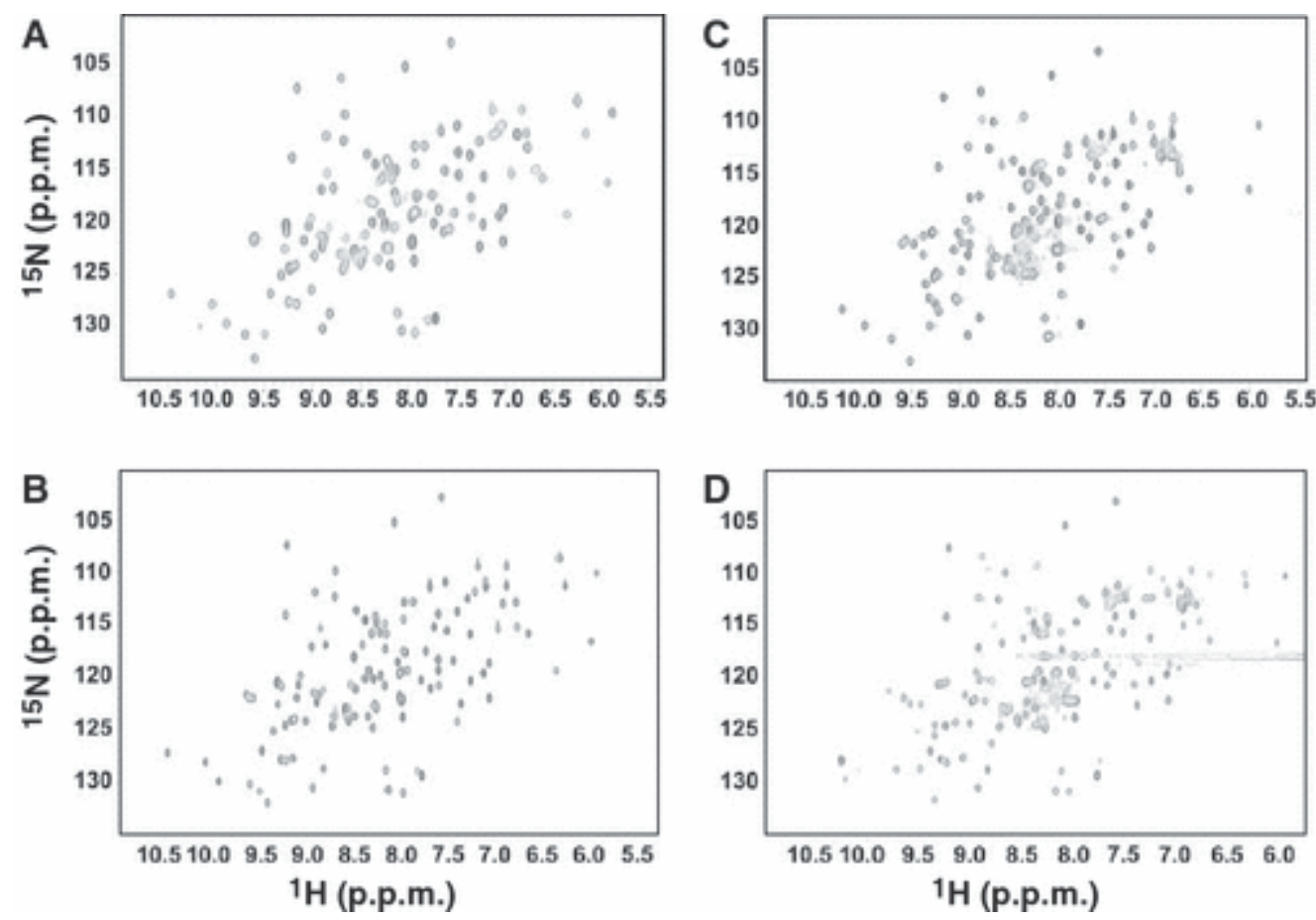

Figure 2.

Comparison of the HSQC spectra for the four frataxin mutants. (A) D122Y; (B) G130V; (C) W155R; and (D) I154F. The spectra were recorded at $600 \mathrm{MHz}$ and $25^{\circ} \mathrm{C}$.

\section{Conformational dynamics of frataxin mutants: different mutants have different tendencies to aggregate}

The NMR spectra for the four frataxin mutants are all compatible with folded species, having appreciable peak dispersion (approximately 4 p.p.m. and 30 p.p.m. dispersion in the ${ }^{1} \mathrm{H}$ and ${ }^{15} \mathrm{~N}$ dimensions, respectively) (Fig. 2B-D). This is confirmed by far-UV CD because the spectrum of the mutants is overall identical to that of the wild-type frataxin (not shown). The NMR spectra obtained for W155R and G130V are very similar to that of the wild-type [15], and the spectrum for D122Y shows some local rearrangement of several resonances. The spectrum for I154F is of lower quality, suggesting the presence of a small, but appreciable, population of either degraded or unfolded protein. Accordingly, it was relatively easy to assign the spectra for the W155R, G130V and D122Y mutants from that of the wild-type, whereas the spectrum for I154F could only be tentatively assigned.

$T_{1}$ and $T_{2}$ as well as steady-state ${ }^{1} \mathrm{H}_{-}{ }^{15} \mathrm{~N}$ NOE and correlation times $\left(\tau_{\mathrm{c}}\right)$ were determined and analysed for the wild-type and the mutant frataxins (Table 1 and Fig. 3; see also supplementary Figs S1-S3). Apart from I154, whose resonance is not observable because of overlap, the other mutation sites have average $T_{1} / T_{2}$ and NOE values. We observed a progressive increase of the average $T_{1}$ values, with a concomitant decrease of the average $T_{2}$, which follows the order wildtype < D122Y < G130V < I154F < W155R (see supplementary Doc. S1 and Scheme S1). In agreement, the correlation times extend from 7.5 to $9.2 \mathrm{~ns}$ for W155R (Table 1 and Fig. 3). This strongly suggests that the mutants have a different tendency towards aggregation. Such behaviour is fully consistent with what had been noticed at the protein purification level because expression of frataxin mutants always results in formation of aggregates and inclusion bodies.

Table 1. Relaxation rate constants, NOE and correlation time. $T_{1}$ and $T_{2}$ as well, as steady-state ${ }^{1} \mathrm{H}-{ }^{15} \mathrm{~N}$ NOE and $\tau_{\mathrm{c}}$, were measured for frataxin variants. 
Protein $T_{1}(\mathrm{~ms}) T_{2}(\mathrm{~ms})$ NOE $\tau_{\mathrm{c}}(\mathrm{ns})$

$\begin{array}{lllll}\text { WT } & 544.3 & 106.9 & 0.76 & 7.9 \\ \text { D122Y } & 578.2 & 115.5 & 0.76 & 7.7 \\ \text { G130V } & 634.5 & 98.4 & 0.77 & 7.5 \\ \text { I154F } & 712.1 & 97.7 & 0.75 & 8.5 \\ \text { W155R } & 746.6 & 90.6 & 0.71 & 9.2\end{array}$

\section{$\frac{W T}{s \quad p} \quad \frac{D 122 Y}{s \quad p} \quad \frac{G 130 V}{s \quad p} \quad \frac{\text { I154F }}{s \quad p} \quad \frac{W 155 R}{s \quad p}$}
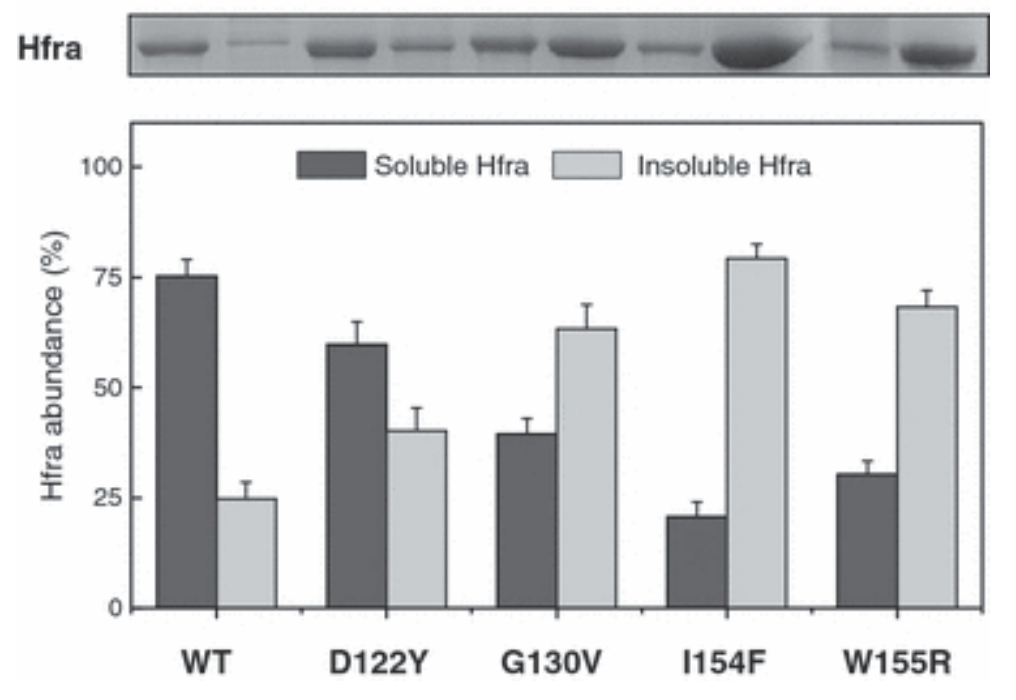

Figure 3.

Representative relaxation parameters of the W155R mutant. The data were collected at $600 \mathrm{MHz}$ and $25^{\circ} \mathrm{C}$. The data for the other mutants are available in the supplementary material.

This was further investigated by carrying out a semi-quantitative analysis of frataxin expression in cell extracts by SDS/PAGE (Fig. 4) and western blot analysis (not shown). Expression systems have been used as a tool to study the foldability and conformational destabilization of human proteins [16], including other mitochondrial proteins [17,18]. The data obtained for the different frataxin variants showed that these have different tendencies to aggregate (Fig. 4). Although wildtype frataxin remains to a considerable extent, and mostly soluble after expression, the same is not observed for the mutant variants. For those, the percentage of frataxin that remains soluble after expression is considerably lower than the fraction that aggregates, and the I154F and the W155R mutants are mostly expressed in an insoluble form (79\% and 68\%, respectively; Fig. 4). This analysis shows that, although all the variants are also found in the soluble fraction, their tendency to misfold in the confined cellular environment could result in an appreciable quantity of aggregated and/or destabilized protein. On the other hand, the average NOE values remain comparable among variants, indicating that the internal flexibility of the protein is essentially invariant. 

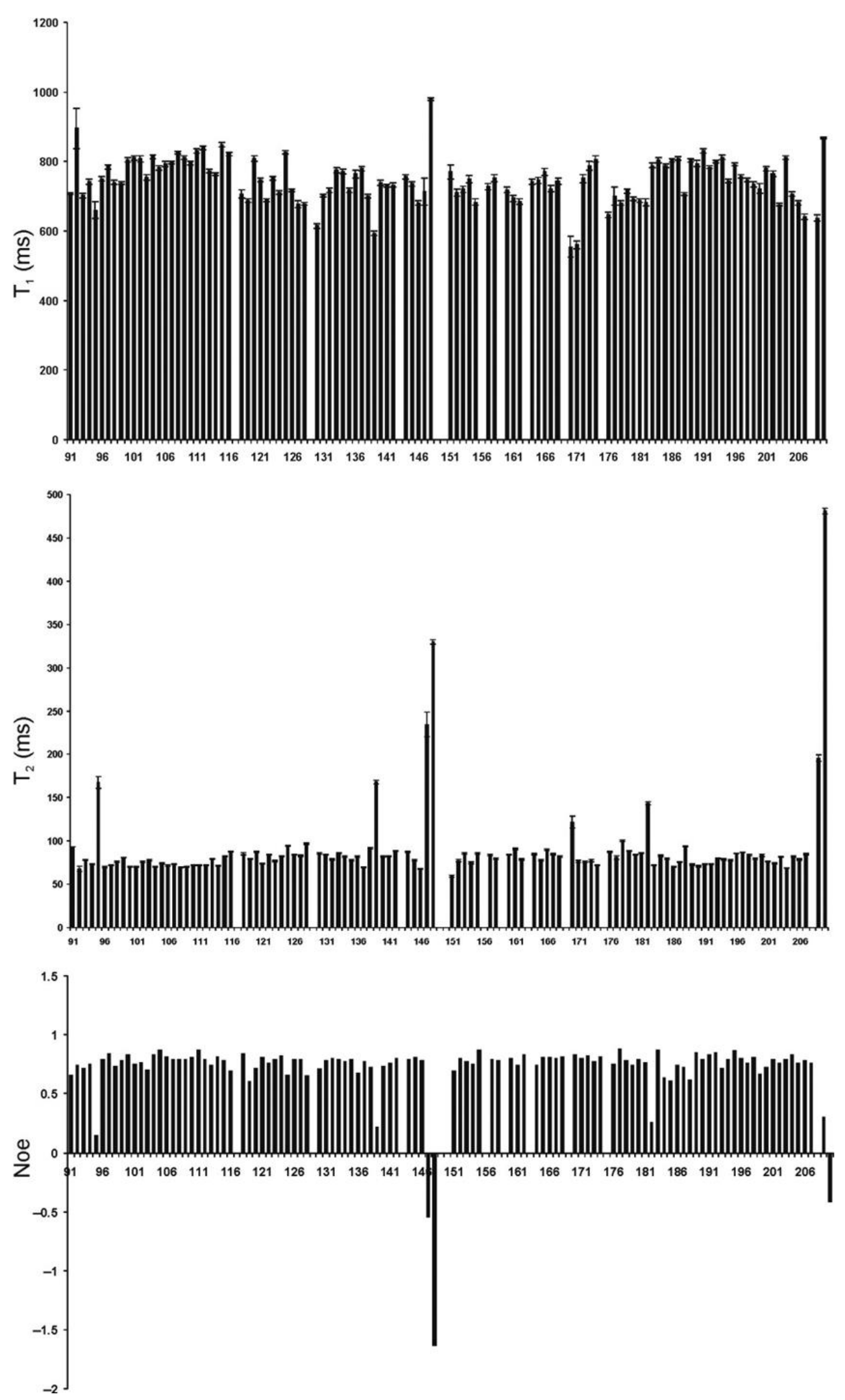

Figure 4 .

Effect of frataxin clinical mutations on the protein aggregation propensity. Top: SDS/PAGE gels obtained from E. coli lysates expressing GST-frataxin fusion proteins $\left(M_{\mathrm{r}}=39.2 \mathrm{kDa}\right)$. Frataxin identity was confirmed by western blot analysis (not shown). For each protein variant, the electrophoretic separations of total protein in both the soluble $(s)$ and insoluble $(p)$ fractions are shown. Bottom: Semi-quantitive analysis of the relative proportion of frataxin present in the soluble and insoluble fractions, obtained from densitometric analysis of gel bands $(n=3)$. 


\section{Probing structural flexibility by limited proteolysis}

Limited proteolysis experiments were used to further identify and characterize the sites of enhanced flexibility or of local unfolding in the frataxin mutants. The rationale for this approach is that chain flexibility is determinant in the proteolytic reaction because digestion of rigid secondary structure elements is extremely disadvantageous thermodynamically [19]. Frataxin nicking reactions were carried out at physiological temperature $\left(37^{\circ} \mathrm{C}\right)$, the reaction products were separated by reverse phase HPLC, and the resulting peptides identified by MS. A comparison of the obtained tryptic maps clearly shows that mutant frataxins are destabilized relatively to the native protein (Fig. 5). All frataxin mutants exhibit an increased proteolytic susceptibility compared to the wild-type, as shown by the higher number of obtained peptides during identical proteolysis periods (Fig. 5). Furthermore, the complexity of the tryptic maps is not identical between mutations: overall, I154F and W155R are more easily accessible to the protease having more degradation sites and peaks, whereas the G130V and D122Y mutants have simpler tryptic maps (Fig. 5). Some additional differences are observed between the mutants, which are suggestive of the local impact that the different mutations have on the protein structure and dynamics. For example, the G130V and D122Y mutations are highly flexible in the loop between strands $\beta 3$ and $\beta 4$, as shown by the appearance of a peak corresponding to the Q153-K164 segment (approximately 36 min; Fig. 5), which is absent in the other mutants. On the other hand, the $\alpha 1$ helix in the I154F and W155R mutants has a decreased rigidity compared to the native protein and the remaining mutants. Proteolysis within a regular secondary structure element such as helices is very unfavourable and does not occur unless some disorder or local breathing is present, as appears to be the case in the I145F and W155R mutants.
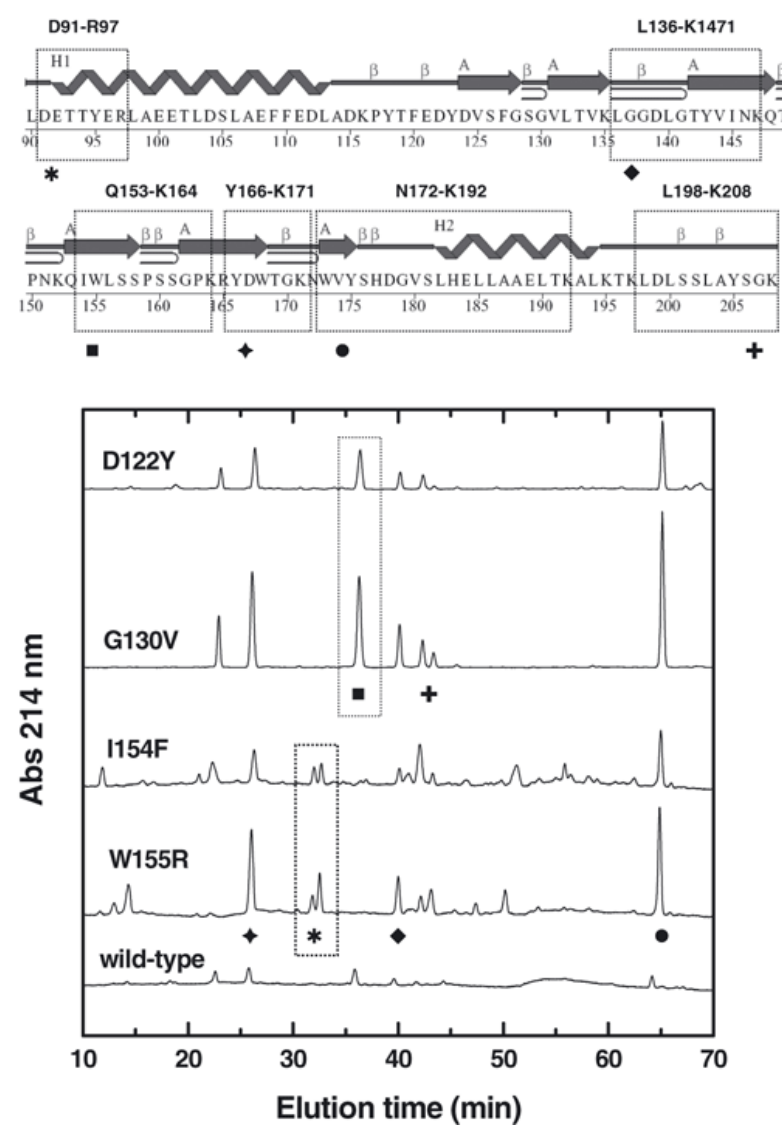

Figure 5 .

\footnotetext{
Trypsin limited proteolysis of frataxin at $\mathrm{pH}$ 8.5. (Top) Secondary structure wiring diagram. The fragments resulting from the tryptic digestion are highlighted by boxes. (Bottom) Peptide maps resulting from partial tryptic digestion: wild-type and mutant variants (D122Y, G130V, I154F and W155R) (data from the wild-type and the last two mutants are redrawn from [9]) after being
} 
incubated with trypsin for $90 \mathrm{~min}$ at $37^{\circ} \mathrm{C}$. Boxes highlight the peaks that are only present on the tryptic digestion of D122Y and G130V or I154F and W155R.

\section{Frataxin mutants have distinct kinetics of proteolytic degradation}

To investigate whether particular regions of frataxin have different degradation rates, we analysed the kinetics of proteolysis of the different frataxin variants (Figs 6 and 7; Table 2). Under the tested conditions, the G130V and D122Y variants are found to undergo proteolysis at higher rates. By contrast, for the I154F and W155R mutants, proteolysis is restricted to particular regions of the protein: fast degradation is observed at cleavage sites within helix $\alpha 1$ (R97), at strand $\beta 5$ (R165) and on the loop between strands $\beta 5$ and $\beta 6$ (K171). The W155R mutant is also cleaved at a faster rate at the protein termini and at the loop between the $\beta 2$ and $\beta 3$ strands, probably as a result of the destabilization of the $\beta 3 / \beta 4$ inter-strand interactions that are affected by this mutation, which is likely to increase the flexibility of the contiguous loop and its cleavability (K135). A comparison between these two mutants suggests that the conformational strain introduced by these mutations results in a more localized destabilization, affecting the stability of the first helix, and eventually perturbing the ridge of negatively charged residues that cluster along the first helix and the first strand, which are known to be involved in iron binding [20].

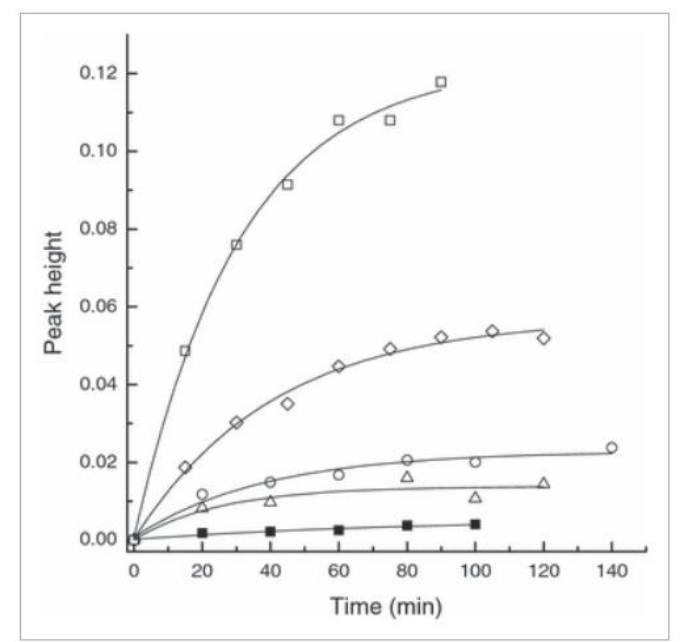

Figure 6.

Time course of trypsin limited proteolysis. The appearance of the peptide eluting at 66 min (Fig. 5) was monitored for wild-type (filled squares) and mutant variants (unfilled diamonds, D122Y; unfilled squares, G130V; unfilled circles, I154F; unfilled triangles, W155R) during incubation with trypsin at $37^{\circ} \mathrm{C}$. Solid traces are fits to first-order reaction rates (wild-type: $k_{\mathrm{obs}}=12.7 \times 10^{-3} \cdot \mathrm{min}^{-1}$; D122Y: $k_{\mathrm{obs}}=32.2 \times 10^{-3} \cdot \mathrm{min}^{-1} ; \mathrm{G} 130 \mathrm{~V}: k_{\mathrm{obs}}=26.4 \times 10^{-3} \cdot \mathrm{min}^{-1}$; I154F: $\left.k_{\mathrm{obs}}=8 \times 10^{-3} \mathrm{~min}^{-1} ; \mathrm{W} 155 \mathrm{R}: k_{\mathrm{obs}}=39.6 \times 10^{-3} \cdot \mathrm{min}^{-1}\right)$. 


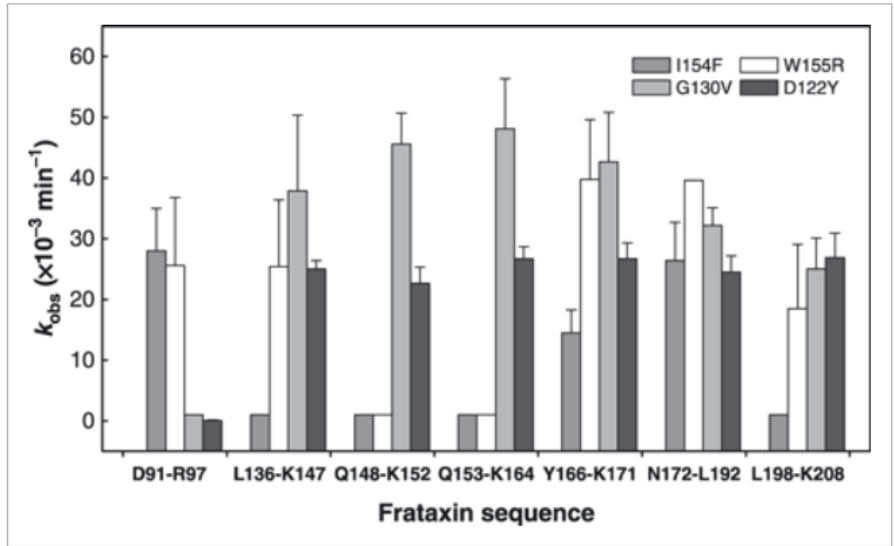

Figure 7.

Comparative plot of proteolysis rates per identified fragment. The observed proteolysis rates of the four frataxin mutant variants are compared for the different digested fragments. The rates determined for native frataxin have been subtracted in each case.

Table 2. Kinetic constants of proteolytic digestion observed for all the identified peaks. Time course of trypsin limited proteolysis: appearance of the peaks with different elution times was monitored for all the proteins under study and the data were fitted to a first-order reaction.

\begin{tabular}{llllll} 
Peak (min) HfrA & \multicolumn{2}{l}{$\boldsymbol{k}_{\text {obs }}\left(\times \mathbf{1 0}^{-\mathbf{3}} \cdot \mathbf{m i n}^{-\mathbf{1}}\right)$} \\
32 & D91-R97 & - & - & $28.1 \pm 8.1$ & $25.6 \pm 11.2$ \\
40 & L136-K147 & $25.0 \pm 1.4$ & $37.9 \pm 12.5$ & & $25.4 \pm 11.0$ \\
23 & L198-K208 & $22.7 \pm 2.6$ & $45.6 \pm 5.1$ & - & - \\
36 & Q153-K164 26.7 & & \\
26 & Y166-K171 $26.7 \pm 2.6$ & $48.1 \pm 8.3$ & - & - \\
66 & N172-K192 $24.5 \pm 2.7$ & $32.2 \pm 2.9$ & $26.4 \pm 6.3$ & $39.6 \pm 21.1$ \\
42 & L198-K208 $26.9 \pm 4.0$ & $25.0 \pm 5.1$ & - & $18.5 \pm 10.6$
\end{tabular}

\section{Impact of different clinical mutations on frataxin stability and iron binding}

To compare the effect of the mutations on the folding thermodynamics of frataxin, we studied their stabilities against chemical unfolding in the presence of urea as measured by far-UV CD and Trp fluorescence emission. As observed for the wild-type protein, the mutant variants show cooperative unfolding transitions (Fig. 8). The results obtained revealed that the two newly studied mutations (D122Y and G130V) are those leading to a higher frataxin destabilization, in agreement with what has been proposed for G130V [21]. The protein stability decreases according to the order: wildtype $>\mathrm{W} 155 \mathrm{R}>\mathrm{I} 154 \mathrm{~F}>\mathrm{D} 122 \mathrm{Y}>\mathrm{G} 130 \mathrm{~V}$ and corresponds to a $\Delta(\Delta \mathrm{G})$ in the range -1.36 to $-2.86 \mathrm{kcal} \cdot \mathrm{mol}^{-1}$ (Table 3). This behaviour was compared with thermal unfolding, as recorded by far-UV CD. We measured the melting curves for G130V and D122Y (Fig. 8) and compared the values with those previously obtained for I154F and W155R [9]. In agreement with the chemical unfolding data, the G130V and D122Y mutants showed the largest variations of melting transitions ( $\Delta \mathrm{T}_{\mathrm{m}}$ of approximately $16{ }^{\circ} \mathrm{C}$ and $23{ }^{\circ} \mathrm{C}$, respectively), while maintaining the reversibility $(>95 \%)$ of the unfolding reaction. 

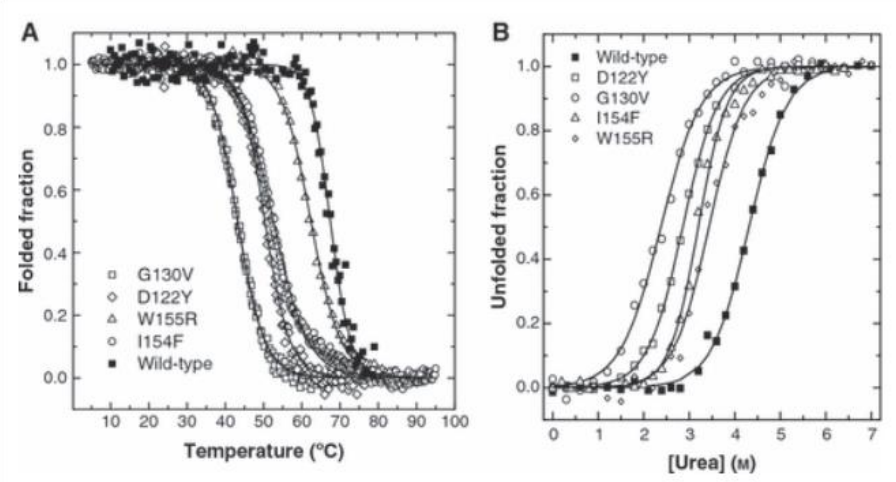

Figure 8.

Thermal (A) and chemical (B) denaturation curves at pH 7.0. filled squares, Wild-type; unfilled diamonds, D122Y; unfilled squares, G130V; unfilled circles, I154F; unfilled triangles, W155R (data from the wild-type and the last two mutants are redrawn from [9]. Lines represent fits to the two-state model [33]; for parameters, see Table 3.

Table 3. Thermodynamic parameters for urea and thermal denaturation of frataxin variants.

\begin{tabular}{|c|c|c|c|c|c|c|}
\hline Protein & $\begin{array}{l}\Delta \mathrm{GH} 2 \mathrm{O} \\
(\mathrm{kcal} \cdot \mathrm{mol}-1)\end{array}$ & $\begin{array}{l}\mathrm{m} \\
(\mathrm{cal} \cdot \mathrm{mol}-1 \cdot \mathrm{m}-1)\end{array}$ & $\begin{array}{l}{[\text { Urea] } 1 / 2} \\
(\mathrm{m})\end{array}$ & $\Delta[$ Urea $] 1 / 2 \mathrm{a}$ & $\begin{array}{l}\Delta(\Delta \mathrm{G}) \\
(\mathrm{cal} \cdot \mathrm{mol}-1) \mathrm{b}\end{array}$ & $\begin{array}{l}\Delta \mathrm{Tm} \\
\left({ }^{\circ} \mathrm{C}\right)\end{array}$ \\
\hline $\begin{array}{l}\text { Wild- } \\
\text { type }^{c}\end{array}$ & $5.6 \pm 0.3$ & $1407 \pm 41$ & 4.3 & & & $66.3 \pm 0.1-$ \\
\hline D122Y & $4.3 \pm 0.2$ & $1498 \pm 23$ & 2.9 & -1.4 & -2110 & $50.4 \pm 0.1-15.9$ \\
\hline G130V & $3.1 \pm 0.3$ & $1310 \pm 60$ & 2.4 & -1.9 & -2863 & $43.2 \pm 0.1-23.1$ \\
\hline $\mathrm{I} 154 \mathrm{~F}^{\mathrm{c}}$ & $5.8 \pm 0.3$ & $1836 \pm 67$ & 3.2 & -1.1 & -1657 & $50.7 \pm 0.1-15.6$ \\
\hline $\mathrm{W} 155 \mathrm{R}^{\mathrm{c}}$ & $5.1 \pm 0.2$ & $1483 \pm 78$ & 3.4 & -0.9 & -1356 & $61.4 \pm 0.4-4.9$ \\
\hline
\end{tabular}

${ }^{\mathrm{a}}$ Difference between the [urea $]_{1 / 2}$ for the wild-type and the mutant forms. ${ }^{\mathrm{b}} \Delta(\Delta \mathrm{G})=\Delta[\text { urea }]_{1 / 2} \times$ average of the three $m$-values [34].

${ }^{\mathrm{c}}$ Data from [9].

The impact of mutations in frataxin was also investigated with respect to its iron-binding properties. Independent experimental evidence suggests that frataxin acts as a cellular iron chaperone and human frataxin has been shown to bind six to seven irons, although with a low affinity [4]. We monitored the iron binding capacity by fluorescence spectroscopy using wild-type frataxin as a control. Under controlled $\mathrm{pH}$ conditions and at $25^{\circ} \mathrm{C}$, the ferric binding capacity of D122Y and G130V appears to be partially impaired; the mutants are only able to bind four irons per molecule (data not shown). As previously reported, the mutants, $1154 \mathrm{~F}$ and $\mathrm{W} 155 \mathrm{R}$, precipitate upon iron binding above the two iron per frataxin threshold [9].

\section{Discussion}

In genetic disorders resulting from missense mutations, the mechanisms by which a single amino acid change triggers disease may result from loss of function, accumulation of toxic species, such as aggregates or amyloid fibres, or dominant negative effects inhibiting the function of the normal protein [22]. In FRDA, the link between a point mutation in frataxin and the disease physiopathology remains unclear, and hypothetical scenarios for the impact of mutations include an 
effect on the folding efficiency, maturation, protein stability, proteolytic susceptibility or function. We have studied different point mutations found in FRDA patients, which are compound heterozygotes for the pathology. These mutations can be grouped according to FRDA symptoms: whereas the I154F and W155R mutations lead to severe FRDA, the mutations G130V and the D122Y account for milder clinical symptoms, although the latter has a very low prevalence [7].

Among these, the most common mutation found in the non-expanded allele is the G130V mutation [7], which was included in the present study. Preliminary work on this mutation has shown that, although human G130V frataxin can complement frataxin-deficient yeast, protein stability is affected and the levels of mature frataxin are diminished [21]. This is in agreement with our findings, which show that this mutation results in a frataxin variant with a decreased conformational stability and iron-binding capacity.

From a structural point of view, our results demonstrate that none of the mutations change significantly the protein fold at room temperature. The heteronuclear single quantum coherence (HSQC) spectra obtained for the frataxin variants are typical of folded species and are very similar to those of wild-type protein. Furthermore, frataxin flexibility is not significantly altered by the insertion of the mutations. Despite retaining the fold, the four mutant variants present a reduced thermodynamic stability, which, in vivo, is likely to cause an increase in the molecular motions and enhance the susceptibility to aggregate and/or to be degraded by the cellular proteases. Limited proteolysis at $37^{\circ} \mathrm{C}$ shows that mutant frataxins have an increased susceptibility towards proteolytic degradation, which is indicative of an enhanced flexibility of the polypeptide chain. A comparison of these results with those obtained by NMR at $25^{\circ} \mathrm{C}$ suggests that raising the temperature to $37{ }^{\circ} \mathrm{C}$ increases the molecular motions enough to allow proteolysis. The increased correlation times measured for the mutants (Table 1) reflect a higher tendency towards aggregation. Furthermore, expression assays revealed that, in mutant frataxins, the soluble/insoluble protein ratio is decreased, and this may play a role in the molecular pathogenesis of FRDA. Accordingly, it has been found that there is an inverse correlation between the level of protein expression and the aggregation rate [23], so that proteins are only marginally soluble to function and aggregation can result from small changes such as chemical modification (e.g. as a consequence of oxidative stress) or genetic mutation (e.g. as in the case of FRDA heterozygous patients).

The observed reduction in iron-binding could also be related to the increased molecular motions. The increased flexibility, combined with the enhanced propensity towards aggregation, could explain why some mutants precipitate upon iron binding (I154F and W155R) or have a lower binding stoichiometry (G130V and D122Y). Under adverse physiological conditions occurring in vivo, such as the oxidative stress observed in FRDA model cells [24-27], these effects could also lead to a perturbation of frataxin structure and dynamics, which could lead to its inactivation or misfolding, further reducing the cellular concentration of functional frataxin.

Altogether, the clinical effects in heterozygous FRDA patients are likely to result from a combination of effects, as observed in other human diseases. For example, in amyotrophic lateral sclerosis, the mutations identified in SOD1 are very different in character, and it has been suggested that the pathology emerges as a result of different reasons or a combination of reasons, from apoprotein destabilization to local unfolding [28]. In the case of FRDA, the results obtained in the present study suggest that factors such as a reduced efficiency of protein folding (resulting in an increase of the aggregation rates), an accelerated degradation in vivo (leading to decreased frataxin levels) and misfolding and conformational destabilization, contribute to a decrease in the levels of functional frataxin. In this scenario, FRDA in heterozygous patients carrying frataxin single point mutations could be considered a type of protein misfolding disorder [22]. 


\section{Experimental procedures}

\section{Chemicals}

All reagents were of the highest purity grade commercially available. The chemical denaturant urea was purchased from Ridel-de Haën (Seelze-Hannover, Germany) and the accurate concentration of the stock solutions in different buffers was confirmed by refractive index measurements.

\section{Protein purification}

All constructs were expressed in Escherichia coli [competent cells BLC21 (DE3); Novagen, EMD Biosciences Inc., San Diego, CA, USA] as fusion proteins with a His-tagged glutathione $S$ transferase (GST) and a cleavage site for tobacco etch virus or PreScission protease (GE Healthcare Bio-Science GmbH, Freiburg, Germany) as previously described [29,30]. The protein concentration was determined using the extinction coefficient $\varepsilon^{280 \mathrm{~nm}}=21930 \mathrm{~m}^{-1} \cdot \mathrm{cm}^{-1}$. As in in previous studies, the protein used corresponded to the conserved C-terminal domain (amino acids 90-210). This form of frataxin has been compared with longer constructs and the mature form, and it has been show that additional residues at the $\mathrm{N}$-terminus are likely unfolded, providing limited information about the protein fold [29]. The mutants were stable in solution, although susceptible to precipitate upon slow freezing. Nevertheless, thawed proteins that had been fast frozen retained their spectroscopic properties and melting temperatures.

\section{SDS/PAGE}

After cell harvesting, $100 \mathrm{mg}$ of cells from each bacterial growth were resuspended in $1.5 \mathrm{~mL}$ of lysis buffer (20 mm Tris- $\mathrm{HCl}, \mathrm{pH} 8,150 \mathrm{~mm} \mathrm{NaCl}, 40 \mathrm{~mm}$ Imidazole, $1 \mathrm{~mm}$ phenylmethanesulfonyl fluoride, DNaseI and lysozyme) and lysed on the French press. After lyses, the samples were centrifuged at $168000 \mathrm{~g}$ for $45 \mathrm{~min}$. The pallet fraction was resuspended in $1.5 \mathrm{~mL}$ of $6 \mathrm{~m} \mathrm{GuHCl}$. The protein concentration of both the pallet and the soluble fraction was determine using Bradford reagent in order to prepare aliquots with the same protein concentration $\left(1 \mathrm{mg} \cdot \mathrm{mL}^{-1}\right)$ to further apply on the gel. A 15\% SDS/PAGE was performed at $200 \mathrm{~V}$ and $25 \mathrm{~mA}$. Proteins were visualized by Coomassie blue staining.

\section{Western blotting}

Proteins separated using SDS/PAGE were transferred from the gel onto poly(vinylidene difluoride) membrane for $1 \mathrm{~h}$ at $45 \mathrm{~mA}$ using a ECL semi-dry blotter (GE Healthcare, Piscataway, NJ, USA). Immunochemical detection of the His-tagged GST frataxin fusion protein was achieved by incubation with anti-GST produced in rabbit (Sigma, St Louis, MO, USA). The antibody was diluted $(1: 1000)$ in $\mathrm{NaCl} / \mathrm{P}_{\mathrm{i}}$-Tween containing nonfat milk. After washing with $\mathrm{NaCl} / \mathrm{P}_{\mathrm{i}}$-Tween, the membrane was incubated with secondary anti-rabbit sera conjugated with horseradish peroxidase (Sigma) and developed with ECL (GE Healthcare).

\section{Spectroscopic methods}

UV/visible spectra were recorded at room temperature in a Shimadzu UVPC-1601 spectrometer (Shimadzu, Kyoto, Japan) equipped with cell stirring. Fluorescence spectroscopy was performed on a Cary Varian Eclipse instrument (Varian NMR, Inc, Palo Alto, CA, USA) $\left(\lambda_{\mathrm{ex}}=280 \mathrm{~nm}\right.$, $\lambda_{\mathrm{em}}=340 \mathrm{~nm}$, slitex $: 5 \mathrm{~nm}$, slitem $: 10 \mathrm{~nm}$, unless otherwise noted) equipped with cell stirring and Peltier temperature control (MJ Research, Watertown, MA, USA). Far-UV CD spectra were 
recorded typically at $0.2 \mathrm{~nm}$ resolution on a Jasco J-715 spectropolarimeter (Jasco Inc., Tokyo, Japan) fitted with a cell holder thermostated equipped with a Peltier.

\section{Trypsin limited proteolysis and LC-MS analysis}

Frataxins were incubated with trypsin (bovine pancreas trypsin, sequencing grade; Sigma) at $37{ }^{\circ} \mathrm{C}$ in $0.1 \mathrm{~m}$ Tris- $\mathrm{HCl}(\mathrm{pH} 8.5)$, in a 100-fold excess over the protease. Aliquots (approximately $0.5 \mathrm{nmol}$ of protein) were sampled at different incubation periods and the reaction stopped by the addition of $0.2 \%(\mathrm{v} / \mathrm{v})$ of trifluoroacetic acid. The products of the proteolysis reaction were analysed by reverse-phase HPLC [9]. The column was regenerated with $0.1 \%(\mathrm{v} / \mathrm{v})$ trifluoroacetic acid. MS analysis was carried out at the ITQB Mass Spectrometry Service Laboratory (Oeiras, Lisbon, Portugal).

\section{Iron-binding assays}

Iron binding stoichiometry was quantitated by iron dependent fluorescence measurements, essentially as described previously [4]. Briefly, tryptophan fluorescence was measured in $1 \mathrm{~mL}$ quartz cuvettes with continuous stirring. The excitation and monitoring wavelengths were 290 and $340 \mathrm{~nm}$, respectively. The binding stoichiometry for ferrous and ferric ion are identical (six or seven irons per frataxin, [4]) and therefore binding of ferric iron was routinely monitored. For the measurements, a $10 \mu \mathrm{m}$ solution of apo frataxin was titrated with ferric ion from a stock solution of $\mathrm{FeCl}_{3}$, over the concentration range $0-120 \mu \mathrm{m}$. The quenching of tryptophan fluorescence induced by the binding of ferric ions was used to calculate the fraction of binding sites occupied. The stoichiometry, $p$, and apparent dissociation constant, $K_{\mathrm{d}}$, were then obtained as previously described by Winzor and Sawyer [31].

\section{NMR spectroscopic methods}

${ }^{15} \mathrm{~N} T_{1}, T_{2}$ and NOE NMR relaxation measurements were performed at $600 \mathrm{MHz}$ and $25{ }^{\circ} \mathrm{C}$ on approximately $0.4 \mathrm{~mm}$ samples. Both $T_{1}$ data and $T_{2}$ data were acquired with ten relaxation delays $(10,100,200,300,400,500,600,700,800,100 \mathrm{~ms}$ and 10, 20, 35, 50, 65, 80, 100, 125, 150, $25 \mathrm{~ms}$, respectively). Experimental steady-state NOE values were determined from the peak intensity ratios of amide signals obtained by recording interleaved 2D Watergate ${ }^{1} \mathrm{H}-{ }^{15} \mathrm{~N}$ HSQC spectra with and without a proton saturation delay of $4 \mathrm{~s}$ and a repetition delay of $4.2 \mathrm{~s} . T_{1}$ and $T_{2}$ relaxation times were obtained by fitting the data with a two-parameter single exponential decay function. The $T_{1}$ and $T_{2}$ values of residues 115, 116, 127, 130, 171, 176, 177, 200, 207, 209 and 210 differ by more than one standard deviation from the mean value and therefore were not considered in the correlation time calculations.

The errors on the $T_{1}$ and $T_{2}$ measurements were estimated to have an average value of $3 \%$, whereas the error on the NOE measurements is approximately $5 \%$. The ${ }^{15} \mathrm{~N}$ heteronuclear relaxation rates were interpreted using the program tensor2 [32]. The internuclear distance $r_{\mathrm{NH}}$ was assumed to be $1.02 \AA$ A. The dipolar and chemical shift anisotropy interactions were assumed to be collinear.

\section{Acknowledgements}

P. Chicau, M. Regalla and A. Coelho from the ITQB Analytical Services Facilities are gratefully acknowledged for their technical contributions. We are also grateful to C. de Chiara for help with analysing the relaxation data. This work was partly supported by a collaborative grant from the Conselho Reitores das Universidades Portuguesas (CRUP, Portugal to C. M. G.) and the British Council (BC, UK to A. P.). A. R. C. is a recipient of a FCT/MCTES PhD fellowship SFRH/BD/24949/2005. 


\section{References}

Delatycki MB, Williamson R \& Forrest SM (2000) Friedreich ataxia: an overview. J Med Genet 37 , $1-8$.

Huynen MA, Snel B, Bork P \& Gibson TJ (2001) The phylogenetic distribution of frataxin indicates a role in iron-sulfur cluster protein assembly. Hum Mol Genet 10, 2463-2468.

3

Muhlenhoff U, Richhardt N, Ristow M, Kispal G \& Lill R (2002) The yeast frataxin homolog Yfh1p plays a specific role in the maturation of cellular Fe/S proteins. Hum Mol Genet 11, 20252036.

4

Yoon T \& Cowan JA (2003) Iron-sulfur cluster biosynthesis. Characterization of frataxin as an iron donor for assembly of [2Fe-2S] clusters in ISU-type proteins. J Am Chem Soc 125, 6078-6084.

5

Chamberlain S, Shaw J, Rowland A, Wallis J, South S, Nakamura Y, von Gabain A, Farrall M \& Williamson R (1988) Mapping of mutation causing Friedreich's ataxia to human chromosome 9. Nature 334, 248-250.

6

Pandolfo M (1999) Molecular pathogenesis of Friedreich ataxia. Arch Neurol 56, 1201-1208.

Cossee M, Durr A, Schmitt M, Dahl N, Trouillas P, Allinson P, Kostrzewa M, Nivelon-Chevallier A, Gustavson KH, Kohlschutter A et al. (1999) Friedreich's ataxia: point mutations and clinical presentation of compound heterozygotes. Ann Neurol 45, 200-206.

8

Campuzano V, Montermini L, Molto MD, Pianese L, Cossee M, Cavalcanti F, Monros E, Rodius F, Duclos F, Monticelli A et al. (1996) Friedreich's ataxia: autosomal recessive disease caused by an intronic GAA triplet repeat expansion. Science 271, 1423-1427.

Correia AR, Adinolfi S, Pastore A \& Gomes CM (2006) Conformational stability of human frataxin and effect of Friedreich's ataxia-related mutations on protein folding. Biochem J 398, 605-611. 
Shan Y, Napoli E \& Cortopassi G (2007) Mitochondrial frataxin interacts with ISD11 of the Nfs1/ISCU complex and multiple mitochondrial chaperones. Hum Mol Genet 16, 929-941.

He Y, Alam SL, Proteasa SV, Zhang Y, Lesuisse E, Dancis A \& Stemmler TL (2004) Yeast frataxin solution structure, iron binding, and ferrochelatase interaction. Biochemistry 43, 16254 16262.

12

Gerber J, Muhlenhoff U \& Lill R (2003) An interaction between frataxin and Isu1/Nfs1 that is crucial for Fe/S cluster synthesis on Isu1. EMBO Rep 4, 906-911.

Lipari GaS A (1982) Model-free approach to the interpretation of nuclear magnetic resonance relaxation in macromolecules. 1 Theory and range of validity. J Am Chem Soc 104, 1546-4559.

14

Maciejewski M, Liu D, Prasad R, Wilson S \& Mullen G (2000) Backbone dynamics and refined solution structure of the N-terminal domain of DNA polymerase beta. Correlation with DNA binding and dRP lyase activity. J Mol Biol 296, 229-253.

\section{5}

Musco G, de Tommasi T, Stier G, Kolmerer B, Bottomley M, Adinolfi S, Muskett FW, Gibson TJ, Frenkiel TA \& Pastore A (1999) Assignment of the 1H, 15N, and 13C resonances of the C-terminal domain of frataxin, the protein responsible for Friedreich ataxia. J Biomol NMR 15, 87-88.

16

Santisteban I, Arredondo-Vega FX, Daniels S \& Hershfield MS (2003) E. coli expression system for identifying folding mutations of human adenosine deaminase. In Protein Misfolding and Disease: Principles and Protocols (Bross P \& Gregersen N, eds). pp. 175-182. Humana Press, Totowa, NJ.

17

Bross P, Jespersen C, Jensen TG, Andresen BS, Kristensen MJ, Winter V, Nandy A, Krautle F, Ghisla S, Bolundi L et al. (1995) Effects of two mutations detected in medium chain acyl-CoA dehydrogenase (MCAD)-deficient patients on folding, oligomer assembly, and stability of MCAD enzyme. J Biol Chem 270, 10284-10290.

\section{8}

Hansen J, Gregersen N \& Bross P (2005) Differential degradation of variant medium-chain acylCoA dehydrogenase by the protein quality control proteases Lon and ClpXP. Biochem Biophys Res Commun 333, 1160-1170. 
Fontana A, de Laureto PP, Spolaore B, Frare E, Picotti P \& Zambonin M (2004) Probing protein structure by limited proteolysis. Acta Biochim Pol 51, 299-321.

Nair M, Adinolfi S, Pastore C, Kelly G, Temussi P \& Pastore A (2004) Solution structure of the bacterial frataxin ortholog, CyaY: mapping the iron binding sites. Structure (Camb) 12, 2037-2048.

Cavadini P, Gellera C, Patel PI \& Isaya G (2000) Human frataxin maintains mitochondrial iron homeostasis in Saccharomyces cerevisiae. Hum Mol Genet 9, 2523-2530.

Gregersen N, Bross P, Vang S \& Christensen JH (2006) Protein misfolding and human disease. Annu Rev Genomics Hum Genet 7, 103-124.

\section{3}

Tartaglia GG, Pechmann S, Dobson CM \& Vendruscolo M (2007) Life on the edge: a link between gene expression levels and aggregation rates of human proteins. Trends Biochem Sci 32, 204-206.

Bulteau AL, Dancis A, Gareil M, Montagne JJ, Camadro JM \& Lesuisse E (2007) Oxidative stress and protease dysfunction in the yeast model of Friedreich ataxia. Free Radic Biol Med 42, 15611570 .

Calabrese V, Lodi R, Tonon C, D’Agata V, Sapienza M, Scapagnini G, Mangiameli A, Pennisi G, Stella AM \& Butterfield DA (2005) Oxidative stress, mitochondrial dysfunction and cellular stress response in Friedreich's ataxia. J Neurol Sci 233, 145-162.

26

Jauslin ML, Meier T, Smith RA \& Murphy MP (2003) Mitochondria-targeted antioxidants protect Friedreich Ataxia fibroblasts from endogenous oxidative stress more effectively than untargeted antioxidants. FASEB J 17, 1972-1974.

Seznec H, Simon D, Bouton C, Reutenauer L, Hertzog A, Golik P, Procaccio V, Patel M, Drapier JC, Koenig M et al. (2005) Friedreich ataxia: the oxidative stress paradox. Hum Mol Genet 14, 463-474. 
Shaw BF \& Valentine JS (2007) How do ALS-associated mutations in superoxide dismutase 1 promote aggregation of the protein? Trends Biochem Sci 32, 78-85.

Musco G, Stier G, Kolmerer B, Adinolfi S, Martin S, Frenkiel T, Gibson T \& Pastore A (2000) Towards a structural understanding of Friedreich's ataxia: the solution structure of frataxin. Structure Fold Des 8, 695-707.

30

Adinolfi S, Nair M, Politou A, Bayer E, Martin S, Temussi P \& Pastore A (2004) The factors governing the thermal stability of frataxin orthologues: how to increase a protein's stability. Biochemistry 43, 6511-6518.

31

Winzor DJ \& Sawyer WH (1995) Quantitative Characterisation of Ligand Binding. Wiley-Liss, New York, NY.

32

Dosset P, Hus JC, Blackledge M \& Marion D (2000) Efficient analysis of macromolecular rotational diffusion from heteronuclear relaxation data. J Biomol NMR 16, $23-28$.

33

Pace CN, Hebert EJ, Shaw KL, Schell D, Both V, Krajcikova D, Sevcik J, Wilson KS, Dauter Z, Hartley RW et al. (1998) Conformational stability and thermodynamics of folding of ribonucleases Sa, Sa2 and Sa3. J Mol Biol 279, 271-286.

34

Pace CN \& Thomson JA (1990) Protein Structure - A Practical Approach. IRL Press, Oxford. 